\title{
Cysteinyl leukotriene levels correlate with 8-isoprostane levels in exhaled breath condensates of atopic and healthy children
}

\author{
Gwo-Hwa Wan' ${ }^{1}$, Dah-Chin Yan ${ }^{2,3,4}$, Hui-Yun Tseng ${ }^{5}$, Tao-Hsin Tung ${ }^{6}$, Syh-Jae Lin ${ }^{4,7}$ and Yu-Wen Lin ${ }^{8}$
}

\begin{abstract}
BACKGROUND: Cysteinyl leukotrienes are important mediators of airway inflammation, whereas 8-isoprostane is a biomarker of oxidative stress. This study evaluated the distributions of cysteinyl leukotriene and 8-isoprostane concentrations in exhaled breath condensates (EBCs) of children. The relationship between cysteinyl leukotriene and 8-isoprostane concentrations in the EBCs was also evaluated.
\end{abstract}

METHODS: The EBCs were collected from 34 children with allergic respiratory diseases and 24 healthy children. All recruited children underwent pulmonary function testing every season. The severity of allergic respiratory diseases and medication status were assessed every month in children with allergic respiratory diseases.

RESULTS: The EBC cysteinyl leukotriene and 8-isoprostane levels were higher in children with asthma and allergic rhinitis than in those with asthma only and healthy children. In asthmatic children, cysteinyl leukotriene and 8-isoprostane levels peaked in the summer. All children showed a clear association between EBC cysteinyl leukotriene and EBC 8-isoprostane levels.

CONCLUSION: The cysteinyl leukotriene and 8-isoprostane concentrations in the EBCs of children significantly varied by season. Oxidative stress correlated with airway inflammation in children.

$\mathbf{T}$ he prevalence of allergic respiratory diseases is gradually increasing. In Taiwan children aged 6-8 years, the prevalence of asthma and allergic rhinitis is $10.4-12.2$ and 24.6-31.4\%, respectively $(1,2)$. In school children, asthma symptoms usually appear in the summer and winter seasons whereas those of allergic rhinitis mainly appear in the winter $(1,2)$. Besides seasonal change, risk factors for allergic respiratory diseases include allergens and air pollutants (3).

As asthma and allergic rhinitis often coexist in the same patient, both conditions might result from the same disease as suggested by the concept of "one airway, one disease" (4). The pathogenesis of asthma and allergic rhinitis in humans mainly involves airway inflammation (3). In vivo, studies show that increased oxidative stress on the respiratory system causes airway obstruction, excess mucus secretion, and airway inflammation $(5,6)$. Hence, airway oxidative stress is a potential indicator of the severity and prognosis of airway inflammation (6). An important biomarker of oxidative stress is 8-isoprostane, which is a prostaglandin-like compound produced in vivo by the free radical-catalyzed peroxidation of arachidonic acid. This compound has been detected in human urine and exhaled breath condensate $(\mathrm{EBC})$ samples $(7,8)$. Concentrations of EBC 8-isoprostane increase with age in healthy adults (9) and are elevated in asthmatic adults $(10,11)$. Concentrations of 8-isoprostane in EBCs are also higher in severe asthmatics as compared with mild and moderate asthmatics and healthy adults $(6,12)$. The EBC 8-isoprostane levels are significantly higher in children with asthma than in healthy children (1316). Another well-documented factor with potential clinical relevance is the seasonality of oxidative stress $(17,18)$.

The pathophysiology of allergic respiratory diseases is characterized mainly by the secretion of inflammatory mediators (histamine, prostaglandin, cytokines, and leukotrienes) by various cell types $(19,20)$. Cysteinyl leukotriene is an eicosanoid derived mainly from linoleic acids. Cysteinyl leukotriene has roles in regulating the biological activities of blood vessels, in tissue repair, and especially in allergic rhinitis. Cysteinyl leukotriene concentrations in EBCs are higher in patients with allergic diseases compared with those in healthy subjects (21). Another study found that EBC levels of cysteinyl leukotriene are higher in patients with moderate or severe asthma compared with those in patients with mild asthma and healthy subjects $(6,22)$. Overall, the results of the studies described earlier agree that variations in the exhaled cysteinyl leukotriene levels are related to airway inflammation in patients with allergic respiratory diseases.

To date, few domestic studies have investigated the distribution of cysteinyl leukotriene and 8-isoprostane concentrations in the EBCs of patients with allergic respiratory diseases, and few have studied the relationship between cysteinyl leukotriene and 8-isoprostane levels in the EBCs. This study therefore

The first two authors contributed equally.

'Department of Respiratory Therapy, College of Medicine, Chang Gung University, Tao-Yuan, Taiwan; ${ }^{2}$ Division of Taipei Pediatrics, Department of Pediatrics, Chang Gung Children's Hospital, Taipei City, Taiwan; ${ }^{3}$ Department of Respiratory Therapy, Chang Gung Memorial Hospital, Taipei City, Taiwan; ${ }^{4}$ College of Medicine, Chang Gung University, Tao-Yuan, Taiwan; ${ }^{5}$ Graduate Institute of Clinical Medical Sciences, Chang Gung University, Tao-Yuan, Taiwan; ${ }^{6}$ Department of Medical Research and Education, Cheng-Hsin General Hospital, Taipei, Taiwan; ${ }^{7}$ Division of Asthma, Allergy, and Rheumatology, Department of Pediatrics, Chang Gung Children's Hospital, Tao-Yuan, Taiwan; ${ }^{8}$ Department of Public Health, College of Medicine, Fu Jen Catholic University, New Taipei City, Taiwan. Correspondence: Gwo-Hwa Wan (ghwan@mail.cgu.edu.tw) 
Table 1. Personal characteristics of the children with allergic respiratory diseases and healthy children

\begin{tabular}{|c|c|c|c|c|c|}
\hline Variable & AS group & AR group & $A S+A R$ group & Control group & $P$ value \\
\hline$n$ & 15 & 9 & 10 & 24 & \\
\hline Age (years) & $10.4 \pm 0.9$ & $8.2 \pm 1.6^{* * * *}$ & $8.8 \pm 1.9^{* * * *}$ & $11.0 \pm 1.3$ & $<0.001$ \\
\hline \multicolumn{6}{|c|}{ Pulmonary function (percentage predicted) } \\
\hline $\mathrm{FEV}_{1}$ & $84.6 \pm 9.3$ & $83.2 \pm 7.9$ & $88.9 \pm 10.2$ & $88.9 \pm 7.9$ & 0.220 \\
\hline MMEF & $76.9 \pm 19.5$ & $87.6 \pm 16.3$ & $66.8 \pm 14.1^{*}$ & $92.2 \pm 16.8$ & 0.001 \\
\hline \multicolumn{6}{|c|}{ Severity of allergic diseases, score } \\
\hline AS & $2.4 \pm 0.9$ & - & $2.8 \pm 1.8$ & & 0.083 \\
\hline AR & - & $2.8 \pm 1.6$ & $2.4 \pm 1.7$ & & 0.375 \\
\hline
\end{tabular}

compared the variations and seasonal changes in exhaled cysteinyl leukotriene and 8-isoprostane levels between children with allergic respiratory disease and healthy children. To elucidate the association between airway inflammation and oxidative stress in humans, the relationship between EBC 8 -isoprostane level and cysteinyl leukotriene concentration in EBCs was also evaluated.

\section{RESULTS}

Table 1 shows that the four groups significantly differed in age $(P<0.01)$ but not in sex. The mean pulmonary function parameters in the children with allergic respiratory diseases were in the normal range. The forced expiratory volume in 1 s/forced vital capacity (percentage predicted) and maximum midexpiratory flow (MMEF) (percentage predicted) significantly differed in the four groups $(P<0.01)$. Specially, the MMEF was much lower in children with asthma and allergic rhinitis $(66.8 \%)$ than in healthy children $(92.2 \%)(P<0.05)$. The mean severity of the allergic respiratory diseases among affected children was at the mild intermittent level. The severity of asthma in the children with asthma and allergic rhinitis did not differ from that in the children with asthma alone. Similarly, the severity of allergic rhinitis did not significantly differ between the children with asthma and allergic rhinitis and the children with only allergic rhinitis.

This study indicated that only $18.3 \%$ of asthmatics regularly used medication, including oral antileukotrienes drugs (montelukast; 6.6\%) and dry powder inhalers containing steroid and long-acting bronchodilator (fluticasone propionate and salmeterol; $11.7 \%)$. In this study, some asthmatic children who did not use medication regularly used a short-acting $\beta_{2}$ agonist when necessary. Approximately $60 \%$ of the children with allergic rhinitis used medication for disease control, including nasal spray drug (fluticasone, budesonide, and triamcinolone acetonide; $58.3 \%$ ), oral antihistamines (cetirizine; $25.0 \%$ ), or a combination of the two drugs (22.2\%). In addition, $72.5 \%$ of the children with both asthma and allergic rhinitis regularly used at least one drug, including nasal spray (67.5\%), oral antileukotrienes (50.0\%), and oral antihistamines $(32.5 \%)$. In

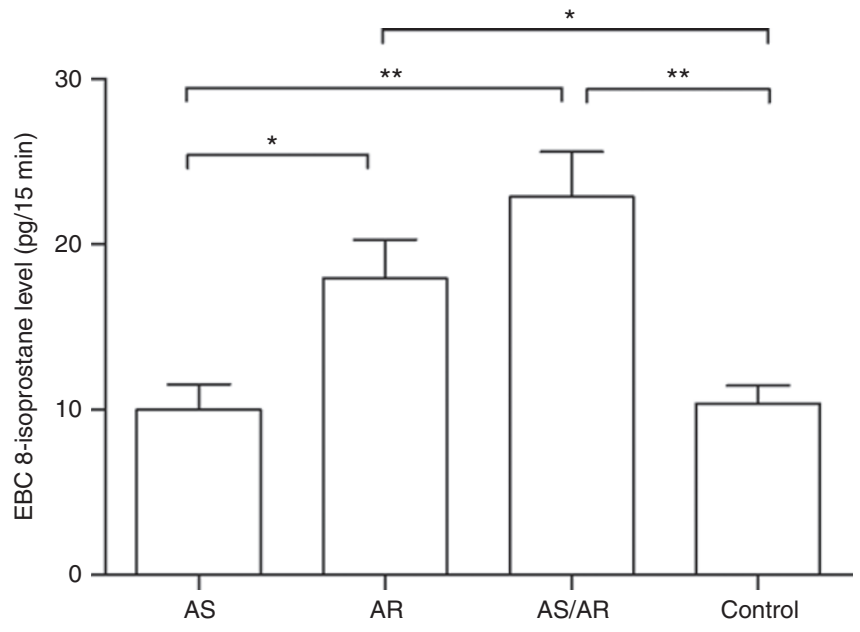

Figure 1. 8-Isoprostane concentration (mean $\pm \mathrm{SE}$ ) in exhaled breath condensates (EBCs) of healthy children $(n=24)$ and children with allergic respiratory diseases (AS, asthma, $n=15 ; A R$, allergic rhinitis, $n=9$; AS $+A R$, $n=10) .{ }^{*} P<0.05$. ${ }^{* *} P<0.01$.

children with mild intermittent allergic respiratory diseases, use of medication did not change during the study period.

Approximately 3\% of EBC 8-isoprostane concentrations were undetectable in the participants of this study. Figure 1 shows that the EBC 8-isoprostane concentration in children with allergic rhinitis (17.94 pg/15 min) was significantly higher than that in both asthmatic children $(10.04 \mathrm{pg} / 15 \mathrm{~min}$; $P=0.023)$ and healthy children $(10.40 \mathrm{pg} / 15 \mathrm{~min} ; P=0.018)$. Moreover, EBC 8-isoprostane concentration in children with asthma and allergic rhinitis $(22.89 \mathrm{pg} / 15 \mathrm{~min})$ was significantly higher than that in asthmatic children $(P<0.001)$ and healthy children $(P<0.001)$. The groups were then evaluated separately for identifying seasonal trends in 8-isoprostane concentrations in the asthmatic children $(6.92 \mathrm{pg} / 15 \mathrm{~min}$ in the spring, $23.62 \mathrm{pg} / 15 \mathrm{~min}$ in the summer, $3.30 \mathrm{pg} / 15 \mathrm{~min}$ in the autumn, and $6.33 \mathrm{pg} / 15 \mathrm{~min}$ in the winter; $F=18.85 ; P<0.01$; Figure $2 \mathrm{a})$, healthy children $(10.75 \mathrm{pg} / 15 \mathrm{~min}$ in the spring, $16.96 \mathrm{pg} / 15 \mathrm{~min}$ in the summer, $5.40 \mathrm{pg} / 15 \mathrm{~min}$ in the autumn, and $8.46 \mathrm{pg} / 15 \mathrm{~min}$ in the winter; $F=7.23 ; P<0.01$; Figure $2 \mathrm{~d}$ ), 


\section{Articles | Wan et al.}
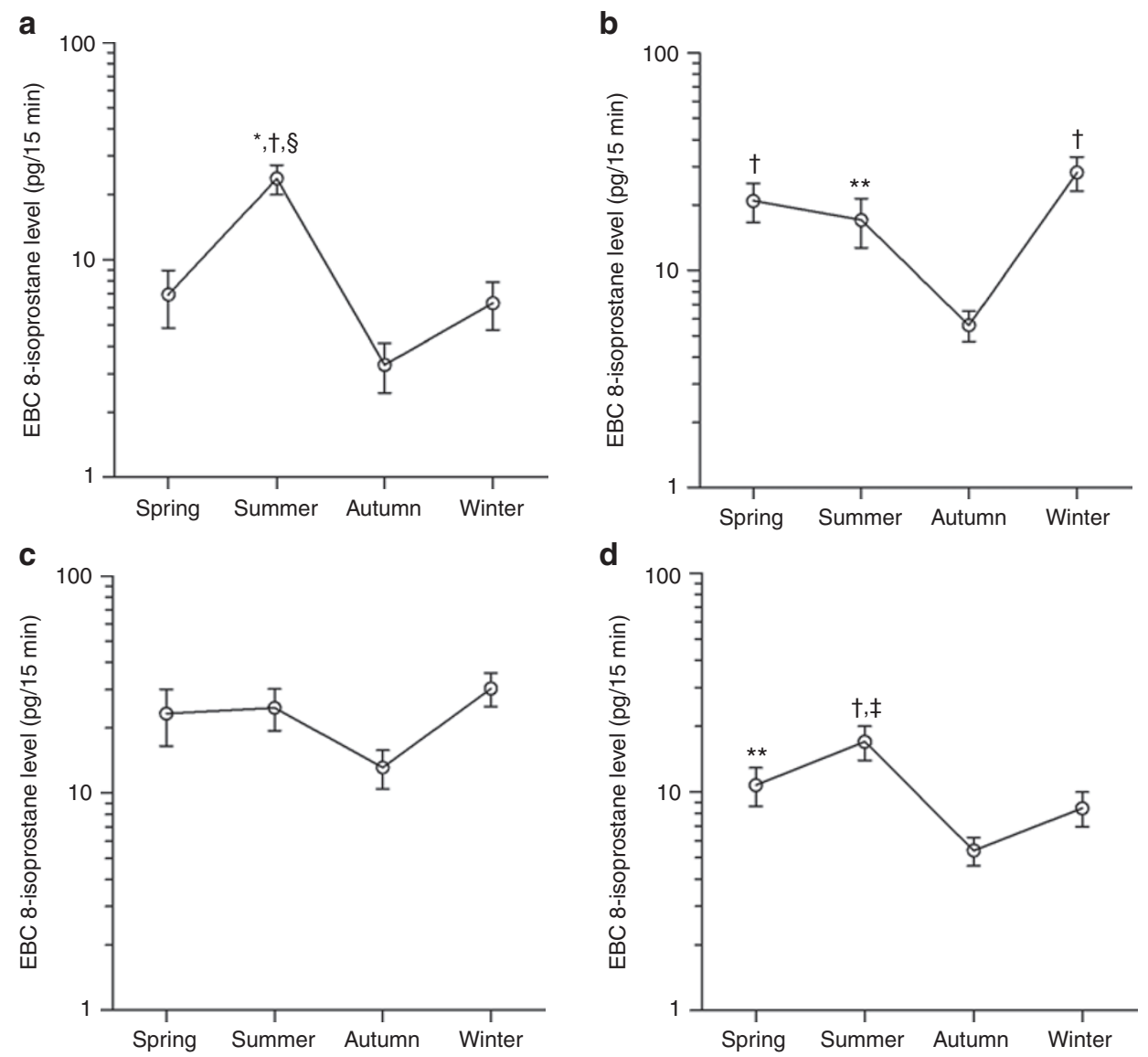

Figure 2. Seasonal variation of 8-isoprostane levels in exhaled breath condensates (EBCs; mean $\pm \mathrm{SE}$ ) in (a) asthmatic children, (b) children with allergic rhinitis, (c) children with asthma and allergic rhinitis, and (d) healthy children. Repeated-measures analysis of variance with Scheffe post hoc test, ${ }^{*} P<0.01$ compared with spring, ${ }^{* *} P<0.05$ compared with autumn, ${ }^{+} P<0.01$ compared with autumn, ${ }^{\ddagger} P<0.05$ compared with winter, and ${ }^{\sharp} P<0.01$ compared with winter.

and children with allergic rhinitis $(20.85 \mathrm{pg} / 15 \mathrm{~min}$ in the spring, $17.06 \mathrm{pg} / 15 \mathrm{~min}$ in the summer, $5.61 \mathrm{pg} / 15 \mathrm{~min}$ in the autumn, and $28.16 \mathrm{pg} / 15 \mathrm{~min}$ in the winter; $F=6.3 ; P<0.01$; Figure 2b). However, Figure $2 \mathrm{c}$ shows that seasonal variation in EBC 8-isoprostane levels was not significant in children with both asthma and allergic rhinitis. The patterns of seasonal variation of EBC 8-isoprostane levels in the allergic rhinitis group also differed from those of the asthma group $(P=0.034)$ and the control group $(P=0.027)$.

This study showed that there was no association between age and exhaled 8-isoprostane concentrations either in healthy children or in those with allergic respiratory diseases. In all the children, EBC 8-isoprostane concentration was weakly associated with MMEF level $(r=-0.15, P=0.02)$. In addition, the EBC 8-isoprostane level was significantly associated with the allergic respiratory disease type ( $\beta=6.50 ; 95 \%$ confidence interval of $\beta=3.67-9.33 ; P<0.01$ ) (data not shown).

The concentration distribution and seasonal variation of EBC cysteinyl leukotriene were similar to those described above for EBC 8-isoprostane. Figure 3 shows that EBC cysteinyl leukotriene concentrations were significantly higher in children with asthma and allergic rhinitis $(251.39 \mathrm{pg} / 15 \mathrm{~min})$ than in asthmatic children $(72.17 \mathrm{pg} / 15 \mathrm{~min} ; P=0.028)$ and

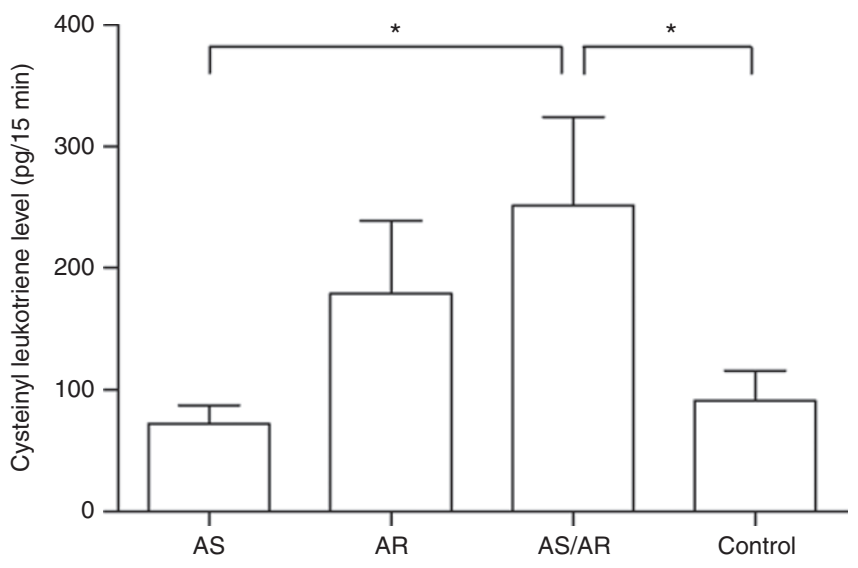

Figure 3. Cysteinyl leukotriene concentration (mean $\pm \mathrm{SE}$ ) in exhaled breath condensates of healthy children $(n=24)$ and those with allergic respiratory diseases (AS, asthma, $n=15 ; \mathrm{AR}$, allergic rhinitis, $n=9$; AS $+A R$, $n=10) . * P<0.05$

healthy children ( $91.46 \mathrm{pg} / 15 \mathrm{~min}, P=0.036)$. Similar seasonal trends in cysteinyl leukotriene concentrations were observed in asthmatic children $(41.34 \mathrm{pg} / 15 \mathrm{~min}$ in the spring, 208.77 $\mathrm{pg} / 15 \mathrm{~min}$ in the summer, $8.60 \mathrm{pg} / 15 \mathrm{~min}$ in the autumn, and $29.99 \mathrm{pg} / 15 \mathrm{~min}$ in the winter; $F=14.236 ; P<0.01$; Figure 4a) 

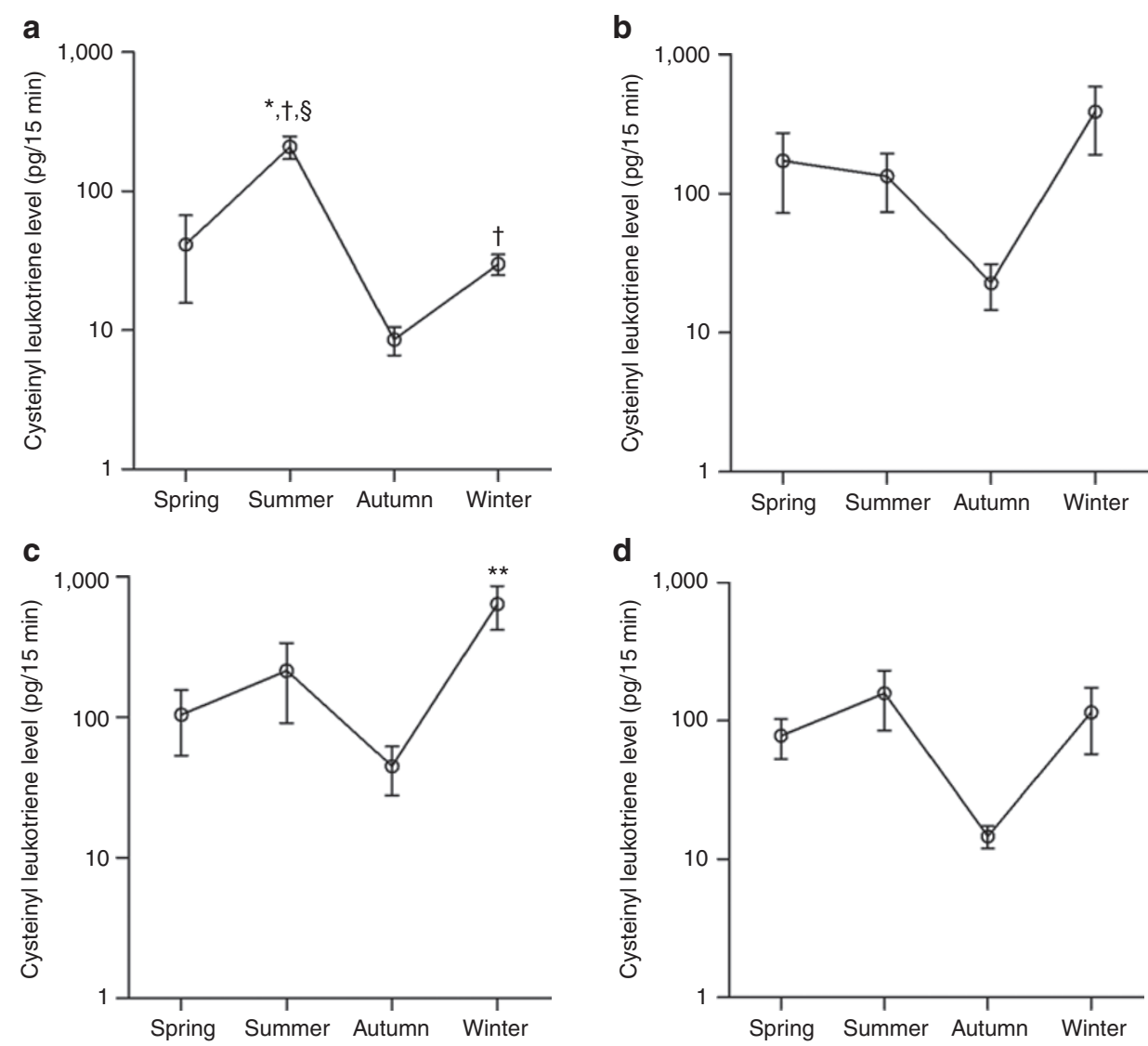

Figure 4. Seasonal variations of cysteinyl leukotriene concentration (mean $\pm \mathrm{SE}$ ) in exhaled breath condensates of (a) asthmatic children, (b) children with allergic rhinitis, (c) children with asthma and allergic rhinitis, and (d) healthy children. Repeated-measures analysis of variance with Scheffe post hoc comparisons, ${ }^{*} P<0.01$ compared with spring, ${ }^{* *} P<0.05$ compared with autumn, ${ }^{\dagger} P<0.01$ compared with autumn, and ${ }^{\S} P<0.01$ compared with winter.

and in children with both asthma and allergic rhinitis $(104.67$ $\mathrm{pg} / 15 \mathrm{~min}$ in the spring, $214.31 \mathrm{pg} / 15 \mathrm{~min}$ in the summer, $44.82 \mathrm{pg} / 15 \mathrm{~min}$ in the autumn, and $641.78 \mathrm{pg} / 15 \mathrm{~min}$ in the winter; $F=4.097$; $P=0.047$; Figure 4c). However, Figure 4b,d shows that no significant seasonal variation in EBC cysteinyl leukotriene levels was observed in children with allergic rhinitis $(F=2.989 ; P=0.114)$ and healthy children $(F=2.144 ; p=$ $0.126)$. Approximately one-sixth of the EBC cysteinyl leukotriene concentrations in all the children reduced below the detection limit of the assay, especially in the EBC samples collected in spring and autumn.

In all the groups, age was not significantly associated with cysteinyl leukotriene concentration. However, Figure 5 shows that cysteinyl leukotriene was positively associated with 8-isoprostane in the asthma group, the allergic rhinitis group, the asthma + allergic rhinitis group, and the control group.

\section{DISCUSSION}

The children in this study had only mild and intermittent allergic respiratory diseases during the study period. However, $53.3 \%$ of the asthma group, $55.5 \%$ of the allergic rhinitis group, and $30 \%$ of the asthma + allergic rhinitis group had abnormal forced expiratory volume in $1 \mathrm{~s}$ levels $(<80 \%$ of the predicted value). The forced expiratory volume in $1 \mathrm{~s} /$ forced vital capacity levels were below the normal value ( $\geq 80 \%$ of the predicted value) in $6.6 \%$ of asthmatic children and in $20 \%$ of children with asthma and allergic rhinitis. In the children with allergic respiratory diseases, $11.1-40 \%$ had abnormal $(<50 \%)$ MMEF levels. The EBC 8-isoprostane level was weakly associated with the MMEF value. To clarify the relationship between EBC 8-isoprostane levels and pulmonary function, further studies are needed to assess larger samples of children with allergic respiratory diseases.

The 8-isoprostane level of healthy children in this study $(10.40 \mathrm{pg} / 15 \mathrm{~min})$ was comparable with that reported in a previous study in England (23). In this study, $12 \%$ of healthy children had elevated EBC 8-isoprostane levels ( $>20 \mathrm{pg} / 15 \mathrm{~min}$ ). Therefore, the general population without airway obstruction and inflammation apparently undergo production of reactive oxygen species and oxidative stress. Of note, the EBC 8-isoprostane level of asthmatic children in this study was drastically lower than that reported in an earlier study of steroid-treated asthmatic children in England (10.04 vs. $33.0 \mathrm{pg} / 15 \mathrm{~min}$, respectively) (23). The different findings might have resulted from the relatively mild and intermittent disease among the asthmatic children in this study. In addition, we found no difference in the EBC 8-isoprostane levels between the asthmatic and the healthy children, which agrees with the results of a previous study in Sweden (24). 


\section{Articles | Wan et al.}

a

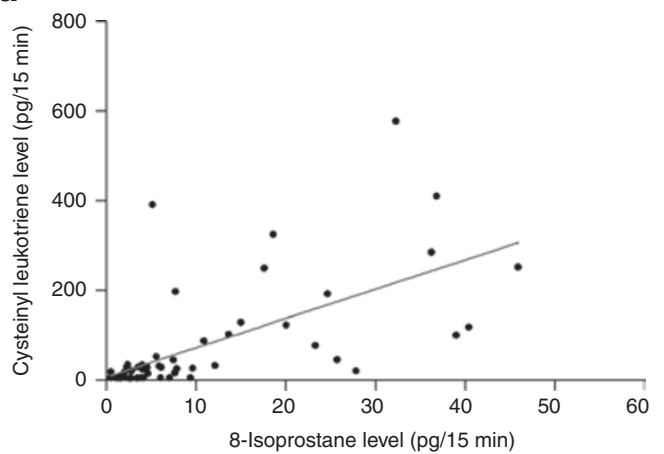

C

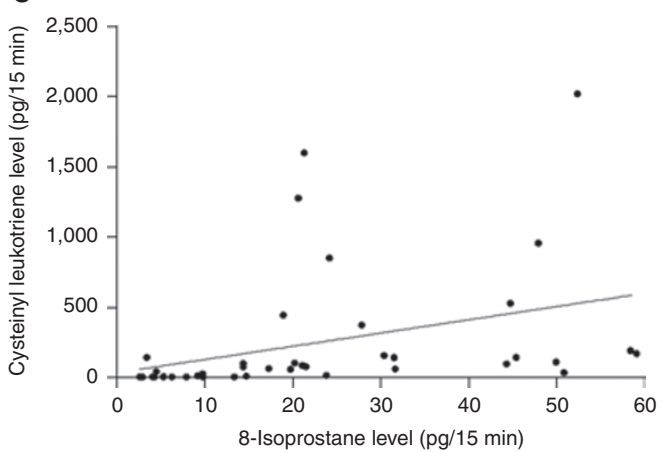

b

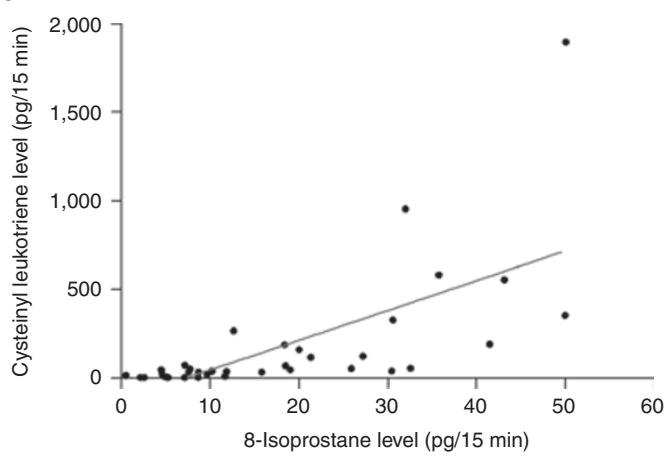

d

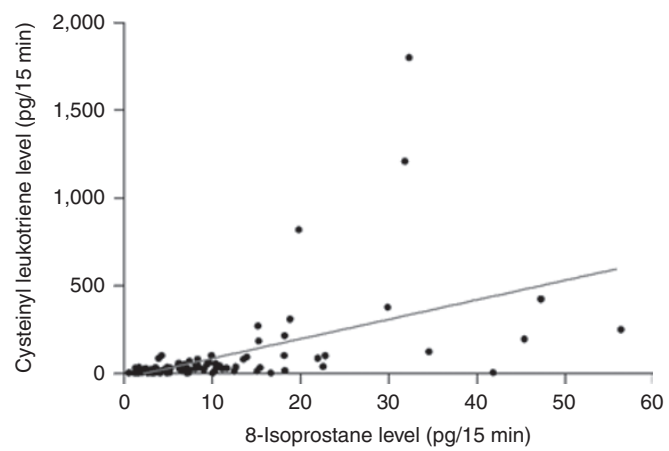

Figure 5. Correlation between cysteinyl leukotriene and 8-isoprostane concentrations in exhaled breath condensates of (a) asthmatic children $(r=0.645$; $P<0.01)$, (b) children with allergic rhinitis $(r=0.663 ; P<0.01)$, (c) children with asthma and allergic rhinitis $(r=0.353 ; P=0.013)$, and (d) healthy children $(r=0.501, P<0.01)$.

Although asthmatic patients are known to have increased EBC cysteinyl leukotriene concentrations $(6,25,26)$, this study shows that cysteinyl leukotriene concentrations in EBCs are similar in healthy and asthmatic children, which is consistent with the results of previous studies in Greece (6) and in the United Kingdom (22). Possible reasons for similar EBC cysteinyl leukotriene levels observed in the asthmatic and healthy children in this study include a mild and intermittent severity of asthma among affected children and the exposure of healthy children to environmental pollutants that may cause mild, persistent airway inflammation and higher concentrations of cysteinyl leukotriene in the EBCs (27-29). In agreement with the results of an Italian study (21), the EBCs of children with asthma and allergic rhinitis had higher cysteinyl leukotriene concentrations compared with the EBCs of healthy children. The observed difference may be related to the activation of nasal cysteinyl leukotriene 1 receptors in patients with allergic rhinitis, which leads to burst releases of cysteinyl leukotriene $(3,6,12,22,30)$. The nasally synthesized cysteinyl leukotrienes collected in EBCs are another potential contributor (21).

In this study, the intraperson reliability of 8 -isoprostane and cysteinyl leukotriene concentrations in the EBC samples showed good agreement, which implies that low intraperson variation would not confound the conclusion regarding the biological relevance of the magnitude of the seasonal variation. Seasonal variations of EBC 8-isoprostane and cysteinyl leukotriene concentrations were evident in both healthy children and in those with allergic respiratory diseases. In both asthmatic children and healthy children, EBC 8-isoprostane levels measured in the summer were higher than those measured in other seasons. This study also revealed higher summertime concentrations of cysteinyl leukotriene in the EBCs of asthmatic children. By contrast, the EBC 8-isoprostane levels of children with allergic rhinitis were highest in the winter and lowest in the autumn. Furthermore, in children with allergic rhinitis and asthma, concentrations of EBC cysteinyl leukotrienes were highest in winter. Of note, the pattern of cysteinyl leukotriene levels in the EBCs of children with allergic respiratory diseases in this study was consistent with those reported elsewhere $(1,2)$. Specifically, the symptoms of asthmatic children appeared most often in the summer, whereas those of allergic rhinitis mainly appeared most often in the winter. As this study did not investigate how seasonal differences in temperature and pollutants affect EBC 8-isoprostane/cysteinyl leukotriene concentrations, further studies are needed to clarify the effects of these environmental factors.

Age was not significantly associated with EBC cysteinyl leukotriene/8-isoprostane levels in children with allergic respiratory disease and healthy children. These results agree with the literature $(13,26)$. Notably, however, all children with allergic respiratory diseases analyzed in this study had a mild and intermittent form of the disease. Therefore, cysteinyl leukotriene levels could not be differentiated according to disease severity. Thus, future studies should assess the relationship between disease severity and cysteinyl leukotriene concentration in the EBCs of children with allergic respiratory diseases. 
Cysteinyl leukotrienes have been implicated in asthma pathophysiology (31). In addition, 8-isoprostane is considered an ideal marker for evaluating oxidative stress in asthma (32). This study showed a significant correlation between cysteinyl leukotriene and 8-isoprostane levels in the EBCs of children with asthma, which is consistent with the reports of a significant association between EBC cysteinyl leukotriene and 8-isoprostane concentrations after treatment with inhaled corticosteroid in children with stable asthma $(r=0.75 ; P<0.01)(33)$ and in asthmatic adults $(r=0.61 ; P<0.0001)(6)$. In this study, both the healthy children and the children with allergic rhinitis, with or without asthma, showed positive associations between EBC cysteinyl leukotriene and 8-isoprostane levels. For undetermined reasons, the association between 8 -isoprostane and cysteinyl leukotriene in EBC samples was slightly stronger in the children with allergic rhinitis than in the asthmatic children. Therefore, further studies are needed for evaluating the relationship among EBC 8-isoprostane/cysteinyl leukotriene levels and the effectiveness of different medications in children with allergic respiratory diseases.

This study had several weaknesses. One limitation is that all EBC samples were concentrated to increase the detectable levels of these EBC biomarkers in children. However, cysteinyl leukotriene concentrations in partial EBC samples were undetectable in the study. In addition, withinday and between-day variability in EBC leukotriene B4 and 8 -isoprostane levels in enzyme immunoassay-based assays had been reported elsewhere (34). Therefore, developing a reproducible, highly sensitive, and highly specific analytical method of measuring low concentrations of EBC biomarkers is essential (35). Differences in EBC mediator concentrations could result from variable production and release of airway droplets. Previous studies indicate that dilutional indicators (e.g., conductivity, urea) should be measured in the lyophilized samples and that EBC biomarkers levels should be corrected with the dilution factor (36-38). However, this study did not measure the dilutional indicator for correction of EBC cysteinyl leukotriene and 8-isoprostane levels, which is a noted limitation. The dilution effect of lyophilized samples on the levels of EBC cysteinyl leukotriene and 8-isoprostane merits further study. In addition, the assay-repeatability affected by lyophilization was not fully elucidated. Therefore, the lyophilization effect on assay-repeatability needs further clarification. The EBCs from children with allergic respiratory diseases were collected under stable conditions in patients without respiratory tract infection and exacerbation in this study. Other clinical uses of EBC 8-isoprostane and cysteinyl leukotriene (e.g., as a supplementary tool for titrating medication, predicting exacerbation, and predicting etiology of exacerbation) also need further study. Finally, additional markers (i.e., nitric oxide, cytokine, and cellular component) in exhaled gas, nasal lavage, or induced sputum are needed for evaluating increases in upper or lower airway inflammation.

In conclusion, $\mathrm{EBC}$ cysteinyl leukotriene and 8-isoprostane levels are effective biomarkers of airway inflammation and oxidative stress status in children. Both cysteinyl leukotriene and 8-isoprostane concentrations in the EBCs of children exhibit significant seasonal variations. In healthy children and in children with allergic respiratory diseases, EBC cysteinyl leukotriene is significantly associated with 8-isoprostane levels.

\section{METHODS}

\section{Study Population}

This study analyzed 34 children (age: 6-13 years) with allergic respiratory diseases, including asthma $(n=15)$, allergic rhinitis $(n=9)$, and asthma and allergic rhinitis $(n=10)$, who were recruited from the Department of Pediatrics of Chang Gung Children Hospital in Taipei, Taiwan. A total of 24 healthy children with no history of allergic or pulmonary disease were also recruited from the same hospital and from the same elementary schools in Taipei city attended by the children with allergic respiratory diseases. All asthmatic subjects were diagnosed by a pediatrician according to the guidelines developed by the Global Initiative for Asthma (27) and by the modified National Asthma Education and Prevention Program (39). A diagnosis of allergic rhinitis was defined according to the Allergic Rhinitis and its Impact on Asthma guidelines (3), excluding those who had a hyperreactive airway response during the bronchodilator inhalation test using methacholine. Children with allergic respiratory diseases were mainly sensitized with house dust mite (Dermatophagoides pteronyssinus and Dermatophagoides farinae) allergens, dog and cat danders, Candida albicans, and cockroach allergen. This study was approved by the Institutional Review Board of Chang Gung Hospital. Before each subject participated in the study, informed written consent was obtained from the family.

\section{Exhaled Cysteinyl Leukotriene and 8-Isoprostane Assays}

In the children with allergic respiratory diseases, antiallergic drugs were not withdrawn from useduring the study period. None of the recruited children had symptoms of airway infection before EBC collection. A noninvasive cooling device (EcoScreen, Jaeger Toennies, Hoechberg, Germany) was used to collect human EBCs in this study. The EBCs from each subject were collected once per season: the spring (February-April), the summer (May-July), the autumn (August-October), and the winter (November-January). To perform EBC sampling, the researcher applied a nose clip on the child and instructed the child to sit straight and breathe normally through a mouthpiece for $15 \mathrm{~min}$. The volume of EBC samples collected from subjects at different time points ranged between 1 and $3 \mathrm{ml}$. After collection, the samples were immediately stored at $-80^{\circ} \mathrm{C}$ until analysis. The EBC samples were pretreated using a lyophilizer model 7750000 (Labconco, Kansas City, MO) and then reconstituted in $0.5 \mathrm{ml}$ of sterile, distilled water. The cysteinyl leukotriene and 8-isoprostane levels in the EBCs were then quantitatively measured by competitive enzyme immunoassays (Cayman Chemical, Ann Arbor, MI) with lower detection limits of 20 and $2.0 \mathrm{pg} / \mathrm{ml}$, respectively. The half value of the low detection limit was used for data analysis if the measured cysteinyl leukotriene and 8-isoprostane levels in the EBC sample fell under the detection limit of the assay, as previously described (21). The original EBC cysteinyl leukotriene and 8-isoprostane concentrations ( $\mathrm{pg} / \mathrm{ml}$ ) were multiplied by $0.5 \mathrm{ml}$ to determine the total amounts (pg) in each sample. Finally, EBC cysteinyl leukotriene and 8-isoprostane levels were presented as $\mathrm{pg} / 15 \mathrm{~min}$ (23). The consistency of the procedures in this study was performed by intraperson reliabilities of 0.96 and 0.942 for cysteinyl leukotriene and 8-isoprostane concentrations in the EBC samples, respectively.

\section{Pulmonary Function Test and Disease Severity Survey}

Each season, a spirometer (MIR Spirolab II, Pinyork, Japan) was used for performing lung function tests, including forced expiratory volume in $1 \mathrm{~s}$, forced vital capacity, and maximum midexpiratory flow, in all children. The severity of allergic respiratory diseases and medication status in the children with allergic respiratory diseases were assessed by a monthly telephone interview. The severity of asthma and allergic 
rhinitis was classified into four levels according to the criteria of the modified National Asthma Education and Prevention Program (39), the frequency and duration of the symptoms, and the medication status (3).

\section{Statistical Analysis}

Statistical analyses were performed using SPSS version 13.0 (SPSS, Chicago, IL). Figures were constructed with GraphPad Prism 5.0 software (GraphPad Software, San Diego, CA). The significancelevel was set at 0.05 . The necessary study sample size was calculated by considering the 8-isoprostane levels in EBC as the primary outcome (23). In addition to 8-isoprostane in exhaled breath, several studies have reported the concentration distributions of EBC leukotrienes B4 and E4 in subjects with allergic respiratory diseases. To the best of our knowledge, however, the distributions of cysteinyl leukotriene levels in asthmatics and healthy controls have not been reported until now. Therefore, the study sample size was calculated according to the assumed criteria in EBC 8-isoprostane with biological significance among groups, that is, the minimal difference in exhaled 8-isoprostane concentrations with biological significance ( $16 \mathrm{pg} / 15 \mathrm{~min})$, a standard deviation of 10 $\mathrm{pg} / 15 \mathrm{~min}$, a type I error of $5 \%$, a power (1-type II error) of $90 \%$, and an estimated dropout rate of $10 \%$ are considered. One-way analysis of variance and two independent samples $t$-test for normally distributed data were used for identifying group differences in continuous variables. A $\chi^{2}$ test was used for identifying group differences in categorical variables. Seasonal variations in EBC cysteinyl leukotriene and 8 -isoprostane levels in children were assessed by repeated-measures analysis of variance with Scheffe post hoc comparisons for normally distributed data. A simple linear regression analysis was constructed for estimating the strength of associations between EBC 8-isoprostane level and category of allergic respiratory disease, with the asthmatic children serving as the reference group. Correlations were performed by Pearson test for normally distributed data to analyze the relationship between the cysteinyl leukotriene and 8-isoprostane concentrations in the EBC samples of all recruited children.

\section{ACKNOWLEDGMENTS}

The authors thank Yueh-Hsiang Chen for her assistance during this investigation.

\section{STATEMENT OF FINANCIAL SUPPORT}

The authors thank the National Science Council of the Republic of China, Taiwan, for financially supporting this research under contracts NSC952314-B-182-056 and NSC96-2314-B-182-017. The funder had no role in study design, data collection and analysis, preparation of the manuscript, or decision to publish.

\section{REFERENCES}

1. Kao CC, Huang JL, Ou LS, See LC. The prevalence, severity and seasonal variations of asthma, rhinitis and eczema in Taiwanese schoolchildren. Pediatr Allergy Immunol 2005;16:408-15.

2. Liao MF, Huang JL, Chiang LC, Wang FY, Chen CY. Prevalence of asthma, rhinitis, and eczema from ISAAC survey of schoolchildren in Central Taiwan. J Asthma 2005;42:833-7.

3. Brozek JL, Bousquet J, Baena-Cagnani CE, et al.; Global Allergy and Asthma European Network; Grading of Recommendations Assessment, Development and Evaluation Working Group. Allergic Rhinitis and its Impact on Asthma (ARIA) guidelines: 2010 revision. J Allergy Clin Immunol 2010;126:466-76.

4. Profita M, Montuschi P, Bonanno A, et al. Novel perspectives in the detection of oral and nasal oxidative stress and inflammation in pediatric united airway diseases. Int J Immunopathol Pharmacol 2010;23:1211-9.

5. Kostikas K, Koutsokera A, Papiris S, Gourgoulianis KI, Loukides S. Exhaled breath condensate in patients with asthma: implications for application in clinical practice. Clin Exp Allergy 2008;38:557-65.

6. Samitas K, Chorianopoulos D, Vittorakis S, et al. Exhaled cysteinyl-leukotrienes and 8-isoprostane in patients with asthma and their relation to clinical severity. Respir Med 2009;103:750-6.

7. Shahid SK, Kharitonov SA, Wilson NM, Bush A, Barnes PJ. Exhaled 8-isoprostane in childhood asthma. Respir Res 2005;6:79.
8. Montuschi P, Ragazzoni E, Valente S, et al. Validation of 8-isoprostane and prostaglandin $\mathrm{E}(2)$ measurements in exhaled breath condensate. Inflamm Res 2003;52:502-7.

9. Cruz MJ, Sánchez-Vidaurre S, Romero PV, Morell F, Muñoz X. Impact of age on $\mathrm{pH}, 8$-isoprostane, and nitrogen oxides in exhaled breath condensate. Chest 2009;135:462-7.

10. Montuschi P, Barnes PJ. Isoprostanes and asthma. Drug Discov Today Ther Strat 2006;3:287-292.

11. Montuschi P, Corradi M, Ciabattoni G, Nightingale J, Kharitonov SA, Barnes PJ. Increased 8-isoprostane, a marker of oxidative stress, in exhaled condensate of asthma patients. Am J Respir Crit Care Med 1999;160:21620.

12. Zhao JJ, Shimizu Y, Dobashi K, et al. The relationship between oxidative stress and acid stress in adult patients with mild asthma. J Investig Allergol Clin Immunol 2008;18:41-5.

13. Baraldi E, Carraro S, Alinovi R, et al. Cysteinyl leukotrienes and 8-isoprostane in exhaled breath condensate of children with asthma exacerbations. Thorax 2003;58:505-9.

14. Carraro S, Cogo PE, Isak I, et al. EIA and GC/MS analysis of 8-isoprostane in EBC of children with problematic asthma. Eur Respir J 2010;35:1364-9.

15. Hasan RA, Thomas J, Davidson B, Barnes J, Reddy R. 8-Isoprostane in the exhaled breath condensate of children hospitalized for status asthmaticus. Pediatr Crit Care Med 2011;12:e25-8.

16. Montuschi P, Mondino C, Koch P, Barnes PJ, Ciabattoni G. Effects of a leukotriene receptor antagonist on exhaled leukotriene $\mathrm{E} 4$ and prostanoids in children with asthma. J Allergy Clin Immunol 2006;118:347-53.

17. Morera AL, Intxausti A, Abreu-Gonzalez P. Winter/summer seasonal changes in malondialdehyde formation as a source of variance in oxidative stress schizophrenia research. World J Biol Psychiatry 2009;10(4 Pt 2):576-80.

18. Konior A, Klemenska E, Brudek M, Podolecka E, Czarnowska E, Beresewicz A. Seasonal superoxide overproduction and endothelial activation in guinea-pig heart; seasonal oxidative stress in rats and humans. J Mol Cell Cardiol 2011;50:686-94.

19. Barnes PJ. Pathophysiology of asthma. Br J Clin Pharmacol 1996;42:3-10.

20. Busse WW, Lemanske RF Jr. Asthma. N Engl J Med 2001;344:350-62.

21. Failla M, Biondi G, Provvidenza Pistorio M, et al. Intranasal steroid reduces exhaled bronchial cysteinyl leukotrienes in allergic patients. Clin Exp Allergy 2006;36:325-30.

22. Csoma Z, Kharitonov SA, Balint B, Bush A, Wilson NM, Barnes PJ. Increased leukotrienes in exhaled breath condensate in childhood asthma. Am J Respir Crit Care Med 2002;166:1345-9.

23. Mondino C, Ciabattoni G, Koch P, et al. Effects of inhaled corticosteroids on exhaled leukotrienes and prostanoids in asthmatic children. J Allergy Clin Immunol 2004;114:761-7.

24. Tufvesson E, Bjermer L. Methodological improvements for measuring eicosanoids and cytokines in exhaled breath condensate. Respir Med 2006;100:34-8.

25. Montuschi P, Barnes PJ. Exhaled leukotrienes and prostaglandins in asthma. J Allergy Clin Immunol 2002;109:615-20.

26. Antczak A, Montuschi P, Kharitonov S, Gorski P, Barnes PJ. Increased exhaled cysteinyl-leukotrienes and 8-isoprostane in aspirin-induced asthma. Am J Respir Crit Care Med 2002;166:301-6.

27. Bateman ED, Hurd SS, Barnes PJ, et al. Global strategy for asthma management and prevention: GINA executive summary. Eur Respir J 2008;31:14378.

28. Canonica GW, Compalati E. Minimal persistent inflammation in allergic rhinitis: implications for current treatment strategies. Clin Exp Immunol 2009;158:260-71.

29. Passalacqua G, Canonica GW. From minimal persistent inflammation to continuous treatment. Eur Respir Dis 2006;34-5.

30. Peters-Golden M, Gleason MM, Togias A. Cysteinyl leukotrienes: multifunctional mediators in allergic rhinitis. Clin Exp Allergy 2006;36:689703.

31. Lewis RA, Austen KF, Soberman RJ. Leukotrienes and other products of the 5-lipoxygenase pathway. Biochemistry and relation to pathobiology in human diseases. N Engl J Med 1990;323:645-55. 
32. Montuschi P, Barnes P, Roberts LJ 2nd. Insights into oxidative stress: the isoprostanes. Curr Med Chem 2007;14:703-17.

33. Zanconato S, Carraro S, Corradi M, et al. Leukotrienes and 8-isoprostane in exhaled breath condensate of children with stable and unstable asthma. J Allergy Clin Immunol 2004;113:257-63.

34. Borrill ZL, Starkey RC, Singh SD. Variability of exhaled breath condensate leukotriene B4 and 8-isoprostane in COPD patients. Int J Chron Obstruct Pulmon Dis 2007;2:71-6.

35. Grob NM, Aytekin M, Dweik RA. Biomarkers in exhaled breath condensate: a review of collection, processing and analysis. J Breath Res 2008;2:037004.
36. Debley JS, Ohanian AS, Spiekerman CF, Aitken ML, Hallstrand TS. Effects of bronchoconstriction, minute ventilation, and deep inspiration on the composition of exhaled breath condensate. Chest 2011;139:16-22.

37. Esther CR Jr, Lazaar AL, Bordonali E, Qaqish B, Boucher RC. Elevated airway purines in COPD. Chest 2011;140:954-60.

38. Lázár Z, Cervenak L, Orosz M, et al. Adenosine triphosphate concentration of exhaled breath condensate in asthma. Chest 2010;138:536-42.

39. Kwok MY, Walsh-Kelly CM, Gorelick MH, Grabowski L, Kelly KJ. National Asthma Education and Prevention Program severity classification as a measure of disease burden in children with acute asthma. Pediatrics 2006;117(4 Pt 2):S71-7. 